\title{
CRITERIA FOR ABSOLUTE CONVERGENCE OF FOURIER SERIES OF FUNCTIONS OF BOUNDED VARIATION
}

\author{
BY \\ INGEMAR WIK( $\left.{ }^{1}\right)$
}

\begin{abstract}
The usual criteria for establishing that a function of bounded variation or an absolutely continuous function has an absolutely convergent Fourier series are given in terms of the modulus of continuity, the integrated modulus of continuity or conditions on the derivative. The relations between these criteria are investigated. A class of functions is constructed to provide counterexamples which show to what extent the existing theorems are best possible. In the case of absolutely continuous functions a few new criteria are given involving the variation of the given function. A couple of necessary and sufficient conditions are given for a class of absolutely continuous functions to have absolutely convergent Fourier series.
\end{abstract}

1. Introduction. We study functions, $f$, with period 1 and their Fourier coefficients

$$
\alpha_{n}=\int_{0}^{1} e^{2 \pi i n x} f(x) d x
$$

$A$ is the class of functions $f$ such that $\sum_{-\infty}^{\infty}\left|\alpha_{n}\right|<\infty$. The modulus of continuity of $f$, $\omega(f, h)$, is defined by

$$
\omega(f, h)=\sup _{\left|x_{1}-x_{2}\right| \leqq h}\left|f\left(x_{1}\right)-f\left(x_{2}\right)\right|
$$

and the integrated modulus of continuity $\omega_{p}(f, h)$ is

$$
\omega_{p}(f, h)=\sup _{0 \leqq t \leqq h}\left(\int_{0}^{1}|f(x+t)-f(x)|^{p}\right)^{1 / p} \text {. }
$$

We state here three basic criteria for a function $f$, of bounded variation (or absolutely continuous), to belong to the class $A$. Theorems I and II are Zygmund's [9, pp. 241-242], while Theorem III originates from Szasz [6]. The last theorem applies to any function of $L_{2}$.

Received by the editors March 18, 1970 and, in revised form, January 13, 1971.

AMS 1969 subject classifications. Primary 4212; Secondary 4210.

Key words and phrases. Absolutely continuous, bounded variation, integrated modulus of continuity.

(1) This paper was written while the author held a fellowship from the AmericanScandinavian Foundation.

Copyright (C) 1972, American Mathematical Society 
THEOREM I. Let f be a continuous periodic function of bounded variation, satisfying

$$
\sum_{n=1}^{\infty} \sqrt{ }\left(\omega\left(f, n^{-1}\right)\right) / n<\infty
$$

Then $f \in A$.

THEOREM II. Let $f$ be an absolutely continuous function with period 1, satisfying

$$
\int_{0}^{1}\left|f^{\prime}\right| \log { }^{+}\left|f^{\prime}\right| d x<\infty
$$

Then $f \in A$.

THEOREM III. Let $f$ be a continuous periodic function, satisfying

$$
\sum_{n=1}^{\infty} \omega_{2}\left(f, n^{-1}\right) / \sqrt{ } n<\infty
$$

Then $f \in A$.

That Theorem I is weaker than Theorem III is an immediate consequence of some properties of moduli of continuity. We shall prove in $\$ 6$ that also Theorem II is weaker than Theorem III. To make the paper self-contained and for references we give the proof of Theorem III.

Proof of Theorem III. Let $c_{n}$ be the Fourier coefficients of $f$. We apply Parseval's relation and get

$$
4 \sum_{-\infty}^{\infty}\left|c_{n}\right|^{2} \sin ^{2} n t \pi=\int_{0}^{1}|f(x+t)-f(x)|^{2} d x
$$

Hence

$$
\sum_{-\infty}^{\infty}\left|c_{n}\right|^{2} \sin ^{2} n h \pi \leqq \omega_{2}^{2}(f, h)
$$

Choosing $h=2^{-p-1}$ we obtain

$$
\sum_{n=2^{p-1}}^{2^{p}}\left|c_{n}\right|^{2} \sin ^{2} \frac{n \pi}{2^{p+1}} \leqq \omega_{2}^{2}\left(f, 2^{-p-1}\right)
$$

and thus

$$
\sum_{n=2^{p-1}}^{2^{p}}\left|c_{n}\right|^{2} \leqq 2 \omega_{2}^{2}\left(f, 2^{-p-1}\right)
$$

By Cauchy's inequality

$$
\sum_{n=2^{p-1}}^{2^{p}}\left|c_{n}\right| \leqq\left(\sum_{2^{p-1}}^{2^{p}}\left|c_{n}\right|^{2} \cdot 2^{p-1}\right)^{1 / 2} \leqq 2^{p / 2} \omega_{2}\left(f, 2^{-p-1}\right) .
$$

If $\sum_{n=1}^{\infty} \omega_{2}\left(f, n^{-1}\right) / \sqrt{ } n<\infty$, then $\sum_{0}^{\infty} 2^{p / 2} \omega_{2}\left(f, 2^{-p}\right)<\infty$ and thus $\sum_{n=1}^{\infty}\left|c_{n}\right|<\infty$.

The same estimations are valid for negative values of $n$ and hence $f \in A$. This completes the proof. 
We want to examine to what extent the three theorems above are the best possible. $\$ 2$ is used for developing some properties of the moduli of continuity and in $\S 3$ we state a few lemmas.

In order to give some counterexamples we construct in $\S 4$ a function, which we will use in the following sections.

Szasz has shown in [6], that condition (1.3) is best possible in the sense that if $\sum_{k=1}^{n} \omega_{2}\left(k^{-1}\right)=O\left(n \omega_{2}\left(n^{-1}\right)\right)$ and $\sum_{1}^{\infty} \omega_{2}\left(n^{-1}\right) / \sqrt{ } n=\infty$, then there exists a function $f \in L_{2}, f \notin A$ such that $\omega_{2}(f, h) \leqq \omega_{2}(h)$.

We show in $\S 5$ that a modified condition (1.3) is the best possible even for absolutely continuous functions.

In $\S 6$ we show that condition (1.2) implies condition (1.3). We also give an example of an absolutely continuous function, satisfying (1.3) but not (1.2).

. It is doubtful that the condition (1.1) is best possible. Salem has in [5] shown that the exponent $\frac{1}{2}$ of $\omega\left(n^{-1}\right)$ cannot be replaced by a greater one. We prove in $\$ 7$ a theorem that contains Salem's theorem as a corollary. For absolutely continuous functions we establish a stronger theorem than Theorem I.

In $\S 8$ we prove criteria when conditions are posed on $\omega_{p}(f, h), p>1$. For further theorems in this case see M. and S. Izumi [2].

There are two functions with the same modulus of continuity and yet one of the functions belongs to $A$ and the other does not. Thus there are no necessary and sufficient conditions that $f \in A$ if we pose conditions on the modulus of continuity only. The only necessary and sufficient condition that applies to a given individual function is given by Zygmund [8]. See also Bari [1, pp. 181-183]. It reads,

THEOREM IV. If the function $f(t)$ satisfies the following conditions

(a) $f$ belongs locally to $A$ for every $t \neq 0$,

(b) $f$ is odd in a neighborhood of zero, $f(0)=0$,

(c) $f^{\prime}$ is nonincreasing in a right neighborhood of zero, then $f \in A$ if and only if

$$
\int_{0}^{1} f^{\prime}(x) \log \frac{1}{x} d x<\infty
$$

We prove in $\S 9$ that the conditions (1.2) and (1.3) are necessary and sufficient for $f$ to belong to $A$, under considerably weaker conditions on $f$, than those of Theorem IV.

2. Moduli of continuity. We will need a few properties of $\omega(f, h)$ and $\omega_{p}(f, h)$, $p \geqq 1$.

(2.1) $\omega_{p}(C f, h)=|C| \omega_{p}(f, h) ; C$ is a constant.

(2.2) $\omega_{p}(f+g, h) \leqq \omega_{p}(f, h)+\omega_{p}(g, h)$.

The latter inequality follows from Minkowski's inequality and the definition of $\omega_{p}$ as a supremum. 
We now restrict ourselves to functions satisfying $f(x+1)-f(x) \equiv C$. Then we have, again using Minkowski's inequality,

$$
\left(\int_{0}^{1}|f(x+2 t)-f(x+t)+f(x+t)-f(x)|^{p} d x\right)^{1 / p} \leqq 2\left(\int_{0}^{1}|f(x+t)-f(x)|^{p} d x\right)^{1 / p} .
$$

This implies

$$
\omega_{p}(f, 2 h) \leqq 2 \omega_{p}(f, h)
$$

Choosing $h=2^{-q-1}$ we obtain

$$
2^{q} \omega_{p}\left(f, 2^{-q}\right) \leqq 2^{q+1} \omega_{p}\left(f, 2^{-q-1}\right) .
$$

If $f$ is real, satisfies $f(x+1)-f(x) \equiv C$ and is monotone, then $a=f(x+2 h)$ $-f(x+h)$ and $b=f(x+h)-f(x)$ have the same sign. Using the inequality $(a+b)^{p}$ $\geqq a^{p}+b^{p}$ we get

$$
\left(\int_{0}^{1}|f(x+2 t)-f(x+t)+f(x+t)-f(x)|^{p} d x\right)^{1 / p} \geqq\left(2 \int_{0}^{1}|f(x+t)-f(x)|^{p} d x\right)^{1 / p}
$$

This implies

$$
\omega_{p}(f, 2 h) \geqq 2^{1 / p} \omega_{p}(f, h),
$$

which for $h=2^{-q-1}$ gives

$$
2^{q} \omega_{p}^{p}\left(f, 2^{-q}\right) \geqq 2^{q+1} \omega_{p}^{p}\left(f, 2^{-q-1}\right) .
$$

In particular for $p=2$ this means that $\left(2^{q / 2} \omega_{2}\left(f, 2^{-q}\right)\right)_{1}^{\infty}$ is a nonincreasing sequence.

If $f$ is of bounded variation, we denote by $V f(x)$ the total variation of $f$ in the interval $[0, x]$. Then $V f$ is nondecreasing and satisfies (2.3) and (2.4), $f$ being replaced by $V f$.

We denote by $V_{f}$ the total variation of $f$ on $[0,1], V f(1)$. Then

$$
\int_{0}^{1}|f(x+t)-f(x)| d x \leqq t V_{f}
$$

which implies

$$
\omega_{1}(f, h) \leqq h V_{f}
$$

Moreover, for $1 \leqq p \leqq 2$,

$$
\int_{0}^{1}|f(x+t)-f(x)|^{2} d x \leqq \omega(f, t)^{2-p} \int_{0}^{1}|f(x+t)-f(x)|^{p} d x
$$

Thus

$$
\omega_{2}(f, h) \leqq(\omega(f, h))^{1-p / 2}\left(\omega_{p}(f, h)\right)^{p / 2} .
$$

In particular, for $p=1$, this yields, using (2.5)

$$
\omega_{2}(f, h) \leqq(\omega(f, h))^{1 / 2}\left(h V_{f}\right)^{1 / 2} .
$$




\section{Some lemmas and a useful function.}

LEMMA 3.1. Let $\sum_{1}^{\infty} a_{n}$ be a divergent positive series with nonincreasing terms. Then $\sum_{1}^{\infty} \min \left(a_{n}, 1 / n\right)$ diverges.

Proof. For any positive series with nonincreasing terms $b_{n}$ we use

$$
2^{p} b_{2^{p+1}} \leqq \sum_{n=2^{p}}^{2^{p+1}} b_{n} \leqq 2^{p} \cdot b_{2^{p}}
$$

to find that

$$
\sum_{1}^{\infty} b_{n}=\infty \Leftrightarrow \sum_{p>1}^{\infty} 2^{p} b_{2^{p}}=\infty
$$

Thus

$$
\sum_{n=1}^{\infty} a_{n}=\infty \Rightarrow \sum_{p=1}^{\infty} 2^{p} a_{2^{p}}=\infty \Rightarrow \sum_{p=1}^{\infty} \min \left(2^{p} a_{2^{p}}, 1\right)=\infty \Rightarrow \sum_{1}^{\infty} \min \left(a_{n}, 1 / n\right)=\infty .
$$

The second implication follows from the fact that either $2^{p} a_{2^{p}}$ is greater than one infinitely many times, in which case the series to the right diverges, or $2^{p} a_{2^{p}}$ is less than one for $p \geqq p_{0}$ in which case the two series are identical for $p \geqq p_{0}$. This proves the lemma.

Lemma 3.2. Let $\sum_{q=1}^{\infty} a_{q}$ be a divergent, positive series, where $a_{q} \leqq 1$. Then, for every $\delta>0$, there exists an increasing sequence of positive numbers $\left(q_{v}\right)_{1}^{\infty}$, such that

$$
(1-\delta)^{q_{v+1}-q_{v}} \leqq \frac{a_{q_{v}}}{a_{q_{v+1}}} \leqq(1+\delta)^{q_{v+1}-q_{v}}
$$

and such that $\sum_{v=1}^{\infty} a_{q_{v}}$ diverges.

A proof of the lemma is given in Wik [7, p. 75].

LEMma 3.3. Let $k$ be an odd, positive integer. Then

$$
S=\left|\sum_{r=0}^{k-1} \exp \left(2 \pi i\left(r n+r^{2}\right) / k\right)\right|=\sqrt{ } k
$$

for all integers $n$.

Proof. A straightforward calculation of $|S|^{2}$.

In the foilowing we shall often use a function $F_{m, k}$, defined by

$F_{m, k}(0)=0$,

$d F_{m, k}(x)=(m / k) \exp \left(i \theta_{r}\right) d x$ on $r / k \leqq x \leqq r / k+1 / m, r=0, \ldots, k-1$,

$d F_{m, k}(x)=0$ elsewhere,

$d F_{m, k}(x+1)=d F_{m, k}(x)$,

$m>k$ are natural numbers, $k$ is odd and $\theta_{r}=2 \pi r^{2} / k$.

The absolutely continuous function $F_{m, k}$ has the following property. 
(3.2) The total variation of $F_{m, k}$ on $[0,1]$ is 1 . The modulus of continuity of $F_{m, k}$ satisfies

$$
\begin{array}{rlrl}
\omega\left(F_{m, k}, 1 / n\right) & \leqq 2 / n, & & n<k, \\
& =1 / k, & & k \leqq n \leqq m, \\
& =m /(n k), & & n>m ; \\
\omega_{2}\left(F_{m, k}, 1 / n\right) \leqq 2 / n, & & n<k, \\
\leqq \sqrt{ } 2 / \sqrt{ }(n k), & & k \leqq n \leqq m, \\
\leqq \sqrt{ }(2 m) / n \sqrt{ } k, & n>m ; \\
\omega_{p}\left(F_{m, k}, 1 / n\right) \leqq 2 / n, & & n<k, \\
\leqq\left(2 k^{1-p} n^{-1}\right)^{1 / p}, & & k \leqq n \leqq m, \\
\leqq 2^{1 / p} m^{1-1 / p} k^{1 / p-1} n^{-1}, & n>m .
\end{array}
$$

We note that with the variation of $F, V F, V F(x)=\int_{0}^{x}|d F(x)|$, we also have the properties (3.2)-(3.5), $F_{m, k}$ being replaced by $V F_{m, k}$.

The Fourier-Stieltjes coefficients of $F_{m, k}$, for convenience written as $F$, satisfy

$$
\begin{aligned}
\left|c_{n}\right|= & \left|\int_{0}^{1} \exp (2 \pi i n x) d F(x)\right| \\
= & (m / k)\left|\exp \left(i \theta_{0}\right)+\exp \left(i \theta_{1}\right) \exp (2 \pi i n / k)+\cdots \exp \left(i \theta_{k-1}\right) \exp (2 \pi i n(k-1) / k)\right| \\
& \cdot\left|\int_{0}^{1 / m} \exp (2 \pi i n x) d x\right| .
\end{aligned}
$$

From Lemma 3.3 it follows that

$$
\left|c_{n}\right|=\frac{m}{k} \sqrt{ } k\left|\int_{0}^{1 / m} \exp (2 \pi i n x) d x\right| .
$$

Now it is easily seen that

$$
\begin{aligned}
1 /(2 \sqrt{ } k) & \leqq\left|c_{n}\right| \leqq 1 / \sqrt{ } k, & & 0 \leqq n \leqq m / 2 \\
0 & \leqq\left|c_{n}\right| \leqq m /(n \sqrt{ } k), & & n>m / 2
\end{aligned}
$$

We observe that when $n$ is approximately equal to $m$ the three expressions $\sqrt{ }\left(\omega\left(F, n^{-1}\right) / n\right), \omega_{2}\left(F, n^{-1}\right) / \sqrt{ } n$ and $\left|c_{n}\right| / n$ have roughly the same size, namely $1 /(n \sqrt{ } k)$.

This is the reason why the function $F_{m, k}$ turns out to be so useful in the constructions we are going to make.

\section{A counterexample.}

THEOREM 4.1. Let $g$ be a nonincreasing function on $(1, \infty)$, such that $2^{q} g\left(2^{q}\right)$ is nondecreasing, $2^{q / 2} g\left(2^{q}\right)$ is nonincreasing and

$$
\sum_{q=1}^{\infty} 2^{q / 2} g\left(2^{q}\right)=\infty
$$


Then there exists a function $f$, absolutely continuous and with period 1, such that $\omega_{2}(f, h) \leqq C g(1 / h)$ and yet $f \notin A$.

Proof. (Compare also the remark after Theorem 9.1.) We put $g_{1}(t)$ $=\min (g(t), 1 / \sqrt{ } t \log t)$. It is easy to verify that the function $g$ has the same monotonic properties as $g$ and it follows from Lemma 3.1 that the series

$$
\sum_{q=1}^{\infty} 2^{q / 2} g_{1}\left(2^{q}\right)=\sum_{q=1}^{\infty} \min \left(2^{q / 2} g\left(2^{q}\right), \frac{1}{q} \log 2\right)
$$

is divergent. Thus we may, without loss of generality, assume that $g\left(2^{q}\right) \leqq 2^{-q / 2} q^{-1}$.

By hypothesis the terms of the series (4.1) form a nonincreasing sequence and therefore (4.1) diverges if and only if $\sum_{1}^{\infty} \alpha_{q}=\infty$, where $\alpha_{q}=5^{q}\left(2^{5 q}\right)^{1 / 2} g\left(2^{5 q}\right)$.

By Lemma 3.2 there exists for every $\delta>0$ a sequence $\left(q_{v}\right)_{1}^{\infty}$ of natural numbers such that

$$
(1-\delta)^{q_{v+1}-q_{v}} \leqq \frac{\alpha_{q_{v+1}}}{\alpha_{q_{\nu}}} \leqq(1+\delta)^{q_{\nu+1}-q_{v}}
$$

and such that

$$
\sum_{\nu=1}^{\infty} \alpha_{q_{v}}=\infty
$$

We put $m_{v}=2^{5 q_{v}}$ and choose $\delta=\frac{1}{6}$. Then by (4.2)

$$
6^{q_{v}-q_{v+1}} \leqq \frac{\sqrt{ }\left(m_{v+1}\right) g\left(m_{v+1}\right)}{\sqrt{ }\left(m_{v}\right) g\left(m_{v}\right)} \leqq 4^{q_{v}-q_{v+1}}
$$

and (4.3) is equivalent to

$$
\sum_{v=1}^{\infty} \sqrt{ }\left(m_{v}\right) g\left(m_{v}\right) \log m_{v}=\infty
$$

We also note that

$$
m_{v+1} g\left(m_{v+1}\right) / m_{v} g\left(m_{v}\right) \geqq\left(m_{v+1} / m_{v}\right)^{1 / 2} \cdot\left(\frac{1}{6}\right)^{q_{v+1}-q_{v}} \geqq 4 .
$$

We shall now use the function $F_{m, k}$ from $\S 3$. For short we put $F_{m_{v}, k_{v}}=F_{v}$. We examine the absolutely continuous function

$$
F=\sum_{v=1}^{\infty} a_{v} F_{v}
$$

where $a_{v} \sim 1 / \nu^{2}$ and $\sqrt{ }\left(k_{v}\right)=a_{v} /\left(m_{v}^{1 / 2} g\left(m_{v}\right)\right)$.

Since, by (4.4), $\sqrt{ }\left(m_{v}\right) g\left(m_{v}\right)$ is decreasing rapidly, we can choose $k_{v}$ as an odd integer and $1 /(\nu+1)^{2} \leqq a_{\nu} \leqq 1 / \nu^{2}$.

The modulus of continuity $\omega_{2}\left(F, n^{-1}\right)$. Since the modulus of continuity is subadditive, (2.2), and since the series (4.7) converges uniformly, we obtain

$$
\omega_{2}\left(F, n^{-1}\right) \leqq \sum_{v=1}^{\infty} a_{v} \omega_{2}\left(F_{v}, n^{-1}\right)
$$


Using the estimation (3.4), we find for $m_{v-1} \leqq n \leqq m_{v}$

$$
\omega_{2}\left(F, n^{-1}\right) \leqq \sum_{\mu=1}^{\nu-1} \frac{a_{\mu} \sqrt{ }\left(2 m_{\mu}\right)}{n \sqrt{ }\left(k_{\mu}\right)}+\sum_{\mu=\nu}^{\infty} \frac{\sqrt{ }\left(2 a_{\mu}\right)}{\sqrt{ }\left(n k_{\mu}\right)}+\sum_{\mu=\nu}^{\infty} \frac{2 a_{\mu}}{n}=\mathrm{I}+\mathrm{II}+\mathrm{III} .
$$

Now $a_{\mu} \sqrt{ }\left(m_{\mu}\right) / \sqrt{ }\left(k_{\mu}\right)=m_{\mu} g\left(m_{\mu}\right)$ is a sequence which by (4.6) is increasing exponentially, at least as $4^{\mu}$. Furthermore $a_{\mu} / \sqrt{ }\left(k_{\mu}\right)=\sqrt{ }\left(m_{\mu}\right) g\left(m_{\mu}\right)$ and this sequence is decreasing by (4.4) exponentially, at most as $4^{-\mu}$. The terms of the series I and II are thus dominated by their last and first terms respectively. The series III is obviously dominated by I for $\nu \geqq \nu_{0}$ and we obtain

$$
\omega_{2}\left(F, n^{-1}\right) \leqq\left(3 m_{v-1} g\left(m_{v-1}\right)\right) / n+\left(3 \sqrt{ }\left(m_{v}\right) g\left(m_{v}\right)\right) / \sqrt{ } n .
$$

By assumption $2^{q} g\left(2^{q}\right)$ is a nondecreasing sequence. Therefore, if $n=2^{q}, m_{v-1} \leqq 2^{q}$ $\leqq m_{v}, m_{v-1} g\left(m_{v-1}\right) \leqq 2^{q} g\left(2^{q}\right)$. The fact that $2^{q / 2} g\left(2^{q}\right)$ is a nonincreasing sequence gives $\sqrt{ }\left(m_{v}\right) g\left(m_{v}\right) \leqq 2^{q / 2} g\left(2^{q}\right)$. (4.9) thus yields $\omega_{2}\left(F, 2^{-q}\right) \leqq 6 g\left(2^{q}\right)$, for $2^{q} \geqq m_{v_{0}}$.

For an arbitrary $h>0$ we choose $q$ such that $2^{-q-1} \leqq h \leqq 2^{-q}$. Then

$$
\omega_{2}(F, h) \leqq \omega_{2}\left(F, 2^{-q}\right) \leqq 6 g\left(2^{q}\right) \leqq 12 g\left(2^{q+1}\right) \leqq 12 g\left(h^{-1}\right),
$$

for $h \leqq 1 / m_{v_{0}}$. We have proved

$$
\omega_{2}(F, h) \leqq 12 g\left(h^{-1}\right) .
$$

The Fourier-Stieltjes coefficients of $F$. We use the estimations (3.6) and the triangle inequality. For $n$ in the interval $m_{v-1} / 2 \leqq n \leqq m_{v} / 2$ we get

$$
\left|d_{n}\right|=\left|\int_{0}^{1} e^{2 \pi i n x} d F(x)\right| \geqq \frac{a_{v}}{2 \sqrt{ }\left(k_{v}\right)}-\left(\sum_{\mu=1}^{v-1} \frac{m_{\mu} a_{\mu}}{n \sqrt{ }\left(k_{\mu}\right)}+\sum_{v+1}^{\infty} \frac{a_{\mu}}{\sqrt{ }\left(k_{\mu}\right)}\right) .
$$

Using the same properties of $a_{\mu}, k_{\mu}$ and $m_{\mu}$ as above, we find

$$
\left|d_{n}\right| \geqq \frac{1}{3} \sqrt{ }\left(m_{v}\right) g\left(m_{v}\right)-2\left(m_{v-1}\right)^{3 / 2} g\left(m_{v-1}\right) / n .
$$

Estimating with an integral, we obtain

$$
\sum_{n=m_{v-1} / 2}^{m_{v} / 2} \frac{\left|d_{n}\right|}{n} \geqq \frac{1}{3} \sqrt{ }\left(m_{v}\right) g\left(m_{v}\right) \log \frac{m_{v}}{m_{v-1}}-2 \frac{\left(m_{v-1}\right)^{3 / 2} g\left(m_{v-1}\right)}{m_{v-1}-1} .
$$

Since $\sum_{v=1}^{\infty} \sqrt{ }\left(m_{v}\right) g\left(m_{v}\right)$ converges as a geometric series and $\log \left(m_{v} / m_{v-1}\right)$ $\geqq C \log m_{v}$ for some positive constant $C$, we deduce that the series $\sum_{n=1}^{\infty}\left(\left|d_{n}\right| / n\right)$ converges and diverges as $\sum_{v=1}^{\infty} \sqrt{ }\left(m_{v}\right) g\left(m_{v}\right) \log m_{v}$. This series is, however, divergent by (4.5) and thus

$$
\sum_{n=1}^{\infty} \frac{\left|d_{n}\right|}{n}=\infty
$$

Construction of $f$. The function $F$ is absolutely continuous and satisfies the conditions of the theorem except that it is not periodic. We consider the function $f$, defined by

$$
f(x)=F(x)-A x,
$$


where $A$ is a constant, chosen such that $f(0)=f(1)$. Then $f$ has period 1 and

$$
\begin{aligned}
\left|d_{n}^{\prime}\right| & =\left|\int_{0}^{1} e^{2 \pi i n x} f(x) d x\right|=\frac{1}{2 \pi n}\left|\int_{0}^{1} e^{2 \pi i n x} d f(x)\right| \\
& =\frac{1}{2 \pi n}\left|\int_{0}^{1} e^{2 \pi i n x} d F(x)\right|=\frac{\left|d_{n}\right|}{2 \pi n}
\end{aligned}
$$

Thus by (4.11) the series $\sum_{1}^{\infty}\left|d_{n}^{\prime}\right|$ diverges and $f \notin A$.

Moreover, by (2.2) and (4.10)

$$
\omega_{2}(f, h) \leqq \omega_{2}(F, h)+\omega_{2}(A x, h)=\omega_{2}(F, h)+A h \leqq 12 g(h)+A h \leqq 13 g(h),
$$

for $h<h_{0}$. The function $f$ thus has the required properties and the theorem is proved.

REMARK. The estimations for $\omega_{2}(F, h)$ and $\omega_{2}(f, h)$ are valid also for their total variations $V F$ and $V f$ respectively. Thus what we have constructed is in fact a function $f \notin A$, such that

$$
\omega_{2}(f, h) \leqq \omega_{2}(V f, h) \leqq C g\left(h^{-1}\right) .
$$

5. Conditions on $\omega_{2}$. In this section we will state a few consequences of the construction in the previous section.

THEOREM 5.1. If uis a function of boundedvariation, such that $\sum_{n=1}^{\infty} \omega_{2}\left(V u, n^{-1}\right) / \sqrt{ } n$ diverges, then there exists a periodic function $f$, absolutely continuous, and such that

$$
\omega_{2}(V f, h) \leqq C \omega_{2}(V u, h)
$$

and yet $f \notin A$.

Proof. The divergence of $\sum_{n=1}^{\infty} \omega_{2}\left(V u, n^{-1}\right) / \sqrt{ } n$ is equivalent to

$$
\sum 2^{q / 2} \omega_{2}\left(V u, 2^{-q}\right)=\infty \text {. }
$$

Furthermore, $V u$ is a monotonous function and we know by (2.3.a) and (2.4.a) that $2^{q} \omega_{2}\left(V u, 2^{-q}\right)$ and $2^{q / 2} \omega_{2}\left(V u, 2^{-q}\right)$ are nondecreasing and nonincreasing respectively. The theorem now follows from Theorem 4.1 if we put $g\left(h^{-1}\right)$ $=\omega_{2}(V u, h)$. Since

$$
\sum_{n=1}^{\infty} \omega_{2}\left(V f, n^{-1}\right) / \sqrt{ } n<\infty \Rightarrow \sum_{n=1}^{\infty} \omega_{2}\left(f, n^{-1}\right) / \sqrt{ } n<\infty \Rightarrow f \in A,
$$

Theorem 5.1 gives the best possible criterion in terms of $\omega_{2}\left(V f, n^{-1}\right)$. However, there are absolutely continuous functions $f$, satisfying $\sum_{1}^{\infty} \omega_{2}\left(f, n^{-1}\right) \sqrt{ } n<\infty$ and $\sum_{1}^{\infty} \omega_{2}\left(V f, n^{-1}\right) \sqrt{ } n=\infty$ and thus functions $u$ belonging to $A$ by Theorem III and yet satisfying the conditions of Theorem 5.1. Such a function can be constructed in the following way. Let the graph consist of $k_{p}$ adjacent triangles with height $a_{p} / k_{p}$ and base $1 / m_{p}$, followed by another sequence of $k_{p+1}$ triangles, with height $a_{p+1} / k_{p+1}$ and base $1 / m_{p+1}$, at a distance of $k_{p} / m_{p}$ from the preceding triangles, $p=1,2, \ldots$ 
Then some computation shows that if we choose $k_{p} \rightarrow \infty$ and $k_{p}=\log m_{p}$, the series $\sum \omega_{2}\left(u, n^{-1}\right) / \sqrt{ } n$ converges as $\sum_{1}^{\infty} a_{p}$ and the series $\sum \omega_{2}\left(V u, n^{-1}\right) / \sqrt{ } n$ diverges as $\sum_{1}^{\infty} a_{p} k_{p}$. Compare also Theorems 9.1 and 9.2.

In proving Theorem 4.1 we needed the properties:

(5.1) $2^{q} g\left(2^{q}\right)$ is a nondecreasing sequence,

(5.2) $2^{q / 2} g\left(2^{q}\right)$ is a nonincreasing sequence.

The property (5.1) is by (2.3.a) satisfied by all the moduli of continuity $\omega_{p}\left(f, 2^{-q}\right)$. It is, however, an open question whether (5.2) is true for $g\left(2^{q}\right)=\omega_{2}\left(f, 2^{-q}\right)$ if $f$ is absolutely continuous and $q$ sufficiently great.

We introduce a modified modulus of continuity by defining

$$
\omega_{2}^{*}(f, h)=\sqrt{ } h \sup _{t \leqq h} \omega_{2}(f, t) / \sqrt{ } t .
$$

We establish a few properties of $\omega_{2}^{*}$. Obviously $\omega_{2}^{*}(f, h) / \sqrt{ } h$ is nondecreasing, which implies that

(5.3) $2^{q / 2} \omega_{2}^{*}\left(f, 2^{-q}\right)$ is nonincreasing.

Moreover, by $(2.3) \omega_{2}(f, 2 h) \leqq 2 \omega_{2}(f, h)$ and thus

$$
\begin{aligned}
2^{q} \omega_{2}^{*}\left(f, 2^{-q}\right) & =2^{q / 2} \sup _{t \leqq 2^{-q}} \omega_{2}(f, t) / \sqrt{ } t \\
& \leqq 2 \cdot 2^{q / 2} \sup _{t \leqq 2^{-q}} \omega_{2}(f, t / 2) / \sqrt{ } t=2^{q+1} \omega_{2}^{*}\left(f, 2^{-q-1}\right) .
\end{aligned}
$$

Therefore,

(5.4) $2^{q} \omega_{2}^{*}\left(f, 2^{-q}\right)$ is nondecreasing.

It is an immediate consequence of the definition of $\omega_{2}^{*}$ that $\omega_{2}(f, h) \leqq \omega_{2}^{*}(f, h)$. We shall see that there is a large class of absolutely continuous functions satisfying

$$
\omega_{2}^{*}(f, h) \leqq C \omega_{2}(f, h)
$$

for some constant $C$, depending on $f$ only.

Lemma 5.2. If $f$ is a monotonous function, then (5.5) holds.

Proof. Choose $q$, such that $2^{-q-1}<h \leqq 2^{-q}$. Then

$$
\omega_{2}^{*}(f, h) \leqq \omega_{2}^{*}\left(f, 2^{-q}\right) .
$$

If $2^{-p-1}<t \leqq 2^{-p}$ we have

$$
\omega_{2}(f, t) / \sqrt{ } t \leqq \sqrt{ } 2 \omega_{2}\left(f, 2^{-p}\right) \cdot 2^{p / 2} .
$$

Thus

$$
\begin{aligned}
\omega_{2}^{*}\left(f, 2^{-q}\right) & =2^{-q / 2} \sup _{t \leqq 2^{-q}} \omega_{2}(f, t) / \sqrt{ } t \\
& \leqq 2 \cdot 2^{-q / 2} \sup _{p \geqq q} \omega_{2}\left(f, 2^{-p}\right) \cdot 2^{p / 2} .
\end{aligned}
$$

By (2.4.a) the right-hand side of (5.7) is equal to $\sqrt{ } 2 \omega_{2}\left(f, 2^{-q}\right)$. Combining (5.6) and (5.7) and using the fact that $2^{q} \omega_{2}\left(f, 2^{-q}\right)$ is nondecreasing, we obtain

$$
\omega_{2}^{*}(f, h) \leqq \sqrt{ } 2 \omega_{2}\left(f, 2^{-q}\right) \leqq 2 \sqrt{ } 2 \omega_{2}\left(f, 2^{-q-1}\right) \leqq 2 \sqrt{ } 2 \omega_{2}(f, h)
$$

which concludes the proof. 
LEMMA 5.3. If

$$
\lim _{h \rightarrow 0+} \omega_{2}(f, h) / \omega_{2}(V f, h)=C_{1}>0,
$$

then (5.5) holds.

Proof. The hypothesis implies that for some constant $C_{2}$ we have

$$
\omega_{2}(V f, h) \leqq C_{2} \omega_{2}(f, h), \quad 0 \leqq h \leqq h_{0} .
$$

Thus for $0 \leqq h<h_{0}$ we have

$$
\omega_{2}^{*}(f, h) \leqq \omega_{2}^{*}(V f, h) \leqq C \omega_{2}(V f, h) \leqq C \cdot C_{2} \omega_{2}(f, h) .
$$

The first inequality follows from the fact that $\omega_{2}(f, t) \leqq \omega_{2}(V f, t)$ and the second from Lemma 5.2. The lemma is thus proved.

Lemma 5.3 obviously includes Lemma 5.2 since for monotonous functions $\omega_{2}(f, h)=\omega_{2}(V f, h)$. It is easy to see that if $f$ is piecewise monotonous and at no point $\bar{D} f=-\underline{D} f=\infty$ or if $\operatorname{Re}(f)$ and $\operatorname{Im}(f)$ have that property, then

$$
\lim _{h \rightarrow 0+} \omega_{2}(f, h) / \omega_{2}(V f, h)=1 .
$$

Another case when $\omega_{2}$ and $\omega_{2}^{*}$ are equivalent is the following.

LEMMA 5.4. If $g(x)=f(x)+A x$ is monotonous for some finite number $A$, then (5.5) holds.

Proof. Since $\omega_{2}$ is subadditive, it follows that $\omega_{2}^{*}$ also is subadditive. Hence $\omega_{2}^{*}(f, h) \leqq \omega_{2}^{*}(g, h)+\omega_{2}^{*}(A x, h)=\omega_{2}^{*}(g, h)+A h$. By Lemma 5.2 and since $\omega_{2}(g, h)$ $\geqq C_{3} h$, we obtain $\omega_{2}^{*}(f, h) \leqq C_{4} \omega_{2}(g, h) \leqq C_{5} \omega_{2}(f, h)$.

As a consequence of Theorems III and 4.1 we state the following theorem.

THEOREM 5.6. If $f$ is a periodic function satisfying $\sum_{1}^{\infty} \omega_{2}^{*}\left(f, n^{-1}\right) / \sqrt{ } n<\infty$, then $f \in A$. Conversely, if $u$ is such that $\sum_{1}^{\infty} \omega_{2}^{*}(u, 1 / n) / \sqrt{ } n=\infty$, there exists an absolutely continuous function $f \notin A$ satisfying $\omega_{2}^{*}(f, h) \leqq C \omega_{2}^{*}(u, h)$.

Proof. The first part of the theorem follows from the fact that $\omega_{2} \leqq \omega_{2}^{*}$. The second part follows from Theorem 4.1 since by (5.3) and (5.4), $\omega_{2}^{*}(u, h)$ has the properties required by the function $g$ of that theorem.

REMARK. By Lemmas 5.4 and 5.5 the theorem is true, with $\omega_{2}^{*}$ replaced by $\omega_{2}$ if the function $u$ is such that either $\lim _{h \rightarrow 0+} \omega_{2}(u, h) / \omega_{2}(V u, h)>0$ or $u(x)+A x$ is monotonous for some finite $A$.

6. The condition $\int_{0}^{1}\left|f^{\prime}\right| \log ^{+}\left|f^{\prime}\right| d x<\infty$. In this section we prove that Theorem II imposes stronger conditions on $f$, than does Theorem 5.6. For the proof we need the following lemma.

LEMMA 6.1. Let $f$ be an absolutely continuous function on $[0,1]$,

$$
E_{n}=\left\{x ; 0 \leqq x \leqq 1 \text { and }\left|f^{\prime}(x)\right|>n\right\} \text { and } V_{n}=\int_{E_{n}}\left|f^{\prime}\right| d x .
$$


Then for any $a>1$

$$
\int_{0}^{1}\left|f^{\prime}\right| \log ^{+}\left|f^{\prime}\right| d x<\infty \Leftrightarrow \sum_{q=1}^{\infty} V_{a^{q}}<\infty .
$$

Proof. We observe that $E_{n} \supseteq E_{n+1}, n=1,2, \ldots$, and put $S_{q}=E_{a^{q}} \backslash E_{a^{q+1}}$. Then

$$
\int_{0}^{1}\left|f^{\prime}\right| \log ^{+}\left|f^{\prime}\right| d x=\sum_{q=0}^{\infty} \int_{S_{q}}\left|f^{\prime}\right| \log ^{+}\left|f^{\prime}\right| d x
$$

This series converges and diverges as

$$
\sum_{q=1}^{\infty} q \int_{S_{q}}\left|f^{\prime}\right| d x=\sum_{q=1}^{\infty} q\left(\int_{E_{q^{q}}}\left|f^{\prime}\right| d x-\int_{E_{a^{q}+1}}\left|f^{\prime}\right| d x\right)
$$

We use Abelian transformation on this series and deduce that it converges exactly when $\sum_{q=1}^{\infty} \int_{E_{a}^{q}}\left|f^{\prime}\right| d x$, or in our notation, $\sum_{q=1}^{\infty} V_{a^{q}}$ is convergent.

THEOREM 6.2. If $f$ is an absolutely continuous function with period 1 , then

$$
\int_{0}^{1}\left|f^{\prime}\right| \log ^{+}\left|f^{\prime}\right| d x<\infty \Rightarrow \sum_{n=1}^{\infty} \omega_{2}^{*}\left(f, n^{-1}\right) / \sqrt{ } n<\infty \text {. }
$$

Furthermore, there are functions $f$ such that the series converges and the integral diverges.

REMARK. Since $\omega_{2}^{*} \geqq \omega_{2}$ the theorem remains true if $\omega_{2}^{*}$ is replaced by $\omega_{2}$. This proves that Theorem II is weaker than Theorem III.

Proof. We suppose that $\int_{0}^{1}\left|f^{\prime}\right| \log ^{+}\left|f^{\prime}\right| d x$ converges and normalize $f$, such that $V_{f}=1$. Using the notations from Lemma 6.1 we put

$$
f(x)=g_{N}(x)+h_{N}(x) \quad \text { where } h_{N}(x)=\int_{E_{N} \cap[0, x]} f^{\prime}(t) d t
$$

and

$$
V f=V g_{N}+V h_{N} \quad \text { where } V h_{N}=\int_{E_{N} \cap[0, x]}\left|f^{\prime}(x)\right| d x
$$

By (2.2)

$$
\omega_{2}\left(V f, n^{-1}\right) \leqq \omega_{2}\left(V g_{N}, n^{-1}\right)+\omega_{2}\left(V h_{N}, n^{-1}\right) .
$$

The definition of $V g_{N}$ yields

$$
V g_{N}(x+1 / n)-V g_{N}(x)=\int_{\substack{x \\\left|f^{\prime}\right|<N}}^{x+1 / n}\left|f^{\prime}(t)\right| d t \leqq \frac{N}{n}
$$

Using the fact that the total variation of $g_{N}$ is less than that of $f$, we obtain

$$
\int_{0}^{1}\left|V g_{N}\left(x+\frac{1}{n}\right)-V g_{N}(x)\right|^{2} d x \leqq \frac{N}{n} \int_{0}^{1}\left|V g_{N}\left(x+\frac{1}{n}\right)-V g_{N}(x)\right| d x \leqq \frac{N}{n^{2}} .
$$


Hence

$$
\omega_{2}\left(V g_{N}, n^{-1}\right) \leqq \sqrt{ } N / n .
$$

The same estimation applied to $V h_{N}$ yields

$$
\omega_{2}^{2}\left(V h_{N}, n^{-1}\right) \leqq \omega\left(V h_{N}, n^{-1}\right) \cdot V_{h_{N}} / n,
$$

where $V_{h_{N}}$ is the total variation on $[0,1]$ of $h_{N}$, i.e. $V_{N}$. Obviously $\omega\left(V h_{N}, n^{-1}\right) \leqq V_{N}$ and thus

$$
\omega_{2}\left(V h_{N}, n^{-1}\right) \leqq V_{N} / \sqrt{ } n .
$$

Combining (6.1), (6.2) and (6.4), we obtain $\omega_{2}\left(V f, n^{-1}\right) \leqq\left(\sqrt{ } N / n+V_{N} / \sqrt{ } n\right)$ for every positive value of $N$. In particular,

$$
2^{q / 2} \omega_{2}\left(V f, 2^{-q}\right) \leqq 2^{-q / 2} N^{1 / 2}+V_{N} .
$$

Fix a number $a$ in the interval $1<a<2$ and for each value of $q$ in (6.5) we choose $N\left(=N_{q}\right)=2^{q} / q^{3}$. Then $N_{q}>a^{q}$ for $q \geqq q_{0}$ and thus $V_{N_{q}} \leqq V_{a^{q}}$. We obtain from (6.5)

$$
2^{q / 2} \omega_{2}\left(V f, 2^{-q}\right) \leqq q^{-3 / 2}+V_{a^{q}} .
$$

By Lemma 6.1 then $\sum_{q=1}^{\infty} 2^{q / 2} \omega_{2}\left(V f, 2^{-q}\right)<\infty$, which is equivalent to

$$
\sum_{n=1}^{\infty} \omega_{2}\left(V f, n^{-1}\right) / \sqrt{ } n<\infty
$$

Since $\omega_{2}^{*}(f, h) \leqq \omega_{2}^{*}(V f, h) \leqq C \omega_{2}(V f, h)$, we have proved the first part of the theorem.

To find an example, where the integral diverges and the series converges, we consider the function $F$ of $\S 4$, (4.7).

By (4.8) we obtain, if $a_{v} m_{v}^{1 / 2} k_{v}^{-1 / 2}$ increases exponentially and $a_{v} k_{v}^{-1 / 2}$ decreases exponentially, that

$$
\sum_{n=m_{v-1}}^{m_{v}} \omega_{2}\left(V F, n^{-1}\right) / \sqrt{ } n \leqq C\left(a_{v-1} k_{v-1}^{-1 / 2}+a_{v} k_{v}^{-1 / 2} \log m_{v}\right) .
$$

An easy computation shows that our integral converges and diverges as the series $\sum_{v=1}^{\infty} a_{v} \log m_{v}$. Thus for example $k_{v}=5^{v}, m_{v}=2^{2^{v}}$ and $a_{v}=\nu^{-2}$ will generate a function $f$ as in (4.12) such that $\sum_{1}^{\infty} \omega_{2}^{*}\left(f, n^{-1}\right) / \sqrt{ } n<\infty$, but $\int_{0}^{1}\left|f^{\prime}\right| \log ^{+}\left|f^{\prime}\right| d x=\infty$.

7. Conditions on $\omega$. In this section we shall in some detail study conditions involving $\omega\left(f, n^{-1}\right)$, that imply $f \in A$. The existing theorem here is Theorem I by Zygmund. Since by (2.7)

$$
\omega_{2}\left(f, n^{-1}\right) / \sqrt{ } n \leqq\left(\omega\left(f, n^{-1}\right) V f\right)^{1 / 2} / n
$$

it follows that Theorem I is weaker than Theorem III. The two theorems are not equivalent, not even for absolutely continuous functions as we see in the following theorem. 
THEOREM 7.1. There is a periodic function $f$, absolutely continuous and such that

(a) $\sum_{1}^{\infty} \omega_{2}^{*}\left(f, n^{-1}\right) / \sqrt{ } n<\infty$ and

(b) $\sum_{n=1}^{\infty}\left(\omega\left(f, n^{-1}\right)\right)^{1 / 2} / n=\infty$.

Proof. We construct a function $F$ of the form (4.7) by choosing $a_{v}=\nu^{-2}, k_{v}=3^{2 v}$, $m_{v}=2^{3^{v}}$. Then, by (4.8)

$$
\sum_{n=m_{v-1}}^{m_{v}} \omega_{2}\left(V F, n^{-1}\right) / \sqrt{ } n \leqq C\left(a_{v-1} k_{v-1}^{-1 / 2}+a_{v} k_{v}^{-1 / 2} \log m_{v}\right)=O\left(a_{v}\right) .
$$

Thus $\sum_{1}^{\infty} \omega_{2}\left(V F, n^{-1}\right) / \sqrt{ } n<\infty$.

We construct $f$ from $F$ as in (4.12) and obtain (a).

By (3.3) and the fact that $\omega(f+g, h) \geqq \omega(f, h)-\omega(g, h)$ we have, for $m_{v-1} \leqq n$ $<m_{v}$,

$$
\omega\left(F, n^{-1}\right) \geqq \frac{a_{v}}{k_{v}}-\sum_{\mu=1}^{v-1} \frac{a_{\mu} m_{\mu}}{n k_{\mu}}-\sum_{\mu=v+1}^{\infty} \frac{a_{\mu}}{k_{\mu}}-\sum_{v+1}^{\infty} \frac{2 a_{\mu}}{n} .
$$

An estimation gives $\omega\left(F, n^{-1}\right) \geqq a_{v} /\left(2 k_{v}\right)$ for $n>20 m_{v-1}$. Thus

$$
\sum_{20 m_{v-1}}^{m_{v}}\left(\omega\left(F, n^{-1}\right)\right)^{1 / 2} / n \geqq C\left(a_{v} k_{v}^{-1}\right)^{1 / 2} \cdot \log m_{v}=C \sqrt{ } a_{v}
$$

and therefore the series $\sum_{1}^{\infty}\left(\omega\left(F, n^{-1}\right)\right)^{1 / 2} / n$ diverges as $\sum_{1}^{\infty} \sqrt{ }\left(a_{v}\right)$.

It is an easy task now to see that the series (b) diverges.

It is not known whether Zygmund's Theorem I is the best possible in the sense that there exists an $f \notin A$ and of bounded variation, satisfying $\omega(f, h) \leqq \omega(g, h)$, as soon as $\sum_{1}^{\infty}\left(\omega\left(g, n^{-1}\right)^{1 / 2} / n=\infty\right.$. Salem [5] has shown that there exists such a function $f$, if $\sum_{n=1}^{\infty} \omega\left(g, n^{-1}\right)^{(1+\varepsilon) / 2} / n=\infty$. It might, however, very well be possible that

$$
\sum_{n=1}^{\infty} \frac{\omega\left(f, n^{-1}\right) \log n}{n}<\infty
$$

is a sufficient condition for a periodic function $f$ of bounded variation to belong to $A$. The condition (7.1) is weaker than $\sum_{n=1}^{\infty}\left(\omega\left(f, n^{-1}\right)\right)^{1 / 2} / n<\infty$ as is seen by the following implications:

$$
\begin{aligned}
\sum_{1}^{\infty} \omega\left(n^{-1}\right)^{1 / 2} / n<\infty & \Leftrightarrow \sum_{1}^{\infty}\left(\omega\left(2^{-p}\right)\right)^{1 / 2}<\infty \\
& \Leftrightarrow \sum_{1}^{\infty} 2^{q}\left(\omega\left(2^{-2 q}\right)\right)^{1 / 2}<\infty \Rightarrow \sum_{1}^{\infty} 2^{2 q} \omega\left(2^{-2 q}\right)<\infty \\
& \Leftrightarrow \sum_{1}^{\infty} p \omega\left(2^{-p}\right)<\infty \Leftrightarrow \sum_{1}^{\infty} \frac{\omega\left(n^{-1}\right) \log n}{n}<\infty .
\end{aligned}
$$

The equivalences follow from the inequality

$$
2^{q(k+1)} a_{2^{q+1}} \leqq \sum_{2^{q}}^{2^{q+1}} p^{k} a_{p} \leqq 2^{(q+1)(k+1)} a_{2^{q}}
$$

which is valid if $\left(a_{p}\right)_{1}^{\infty}$ is a nonincreasing sequence. 
The following example shows that we cannot have a weaker condition on a function of bounded variation than (7.1) to be sure that $f \in A$.

THEOREM 7.2. Let $\omega(h)$ be a nondecreasing function such that $\left(2^{p} \omega\left(2^{-p}\right)\right)_{1}^{\infty}$ is a nondecreasing sequence and

$$
\sum_{1}^{\infty} \frac{\omega\left(n^{-1}\right) \log n}{n}=\infty
$$

Then there exists an absolutely continuous function $f \notin A$ such that $\omega(f, h) \leqq \omega(h)$

Proof. Condition (7.2) is equivalent to $\sum_{q=1}^{\infty} 4^{q} \omega\left(2^{-2^{q}}\right)=\infty$. We may, without loss of generality, assume that the terms of this series are uniformly bounded. Using Lemma 3.2 we obtain, for each $\delta>0$, a sequence $\left(q_{v}\right)_{1}^{\infty}$, such that

$$
\sum_{\nu=1}^{\infty} 4^{q_{v} \omega}\left(2^{-2^{q_{v}}}\right)=\sum_{1}^{\infty} \alpha_{\nu}=\infty
$$

and

$$
(1-\delta)^{q_{v+1}-q_{v}} \leqq \frac{\alpha_{v+1}}{\alpha_{v}} \leqq(1+\delta)^{q_{v+1}-q_{v}}
$$

We fix a $\delta<\frac{1}{6}$. It is easy to see that there exists a nonincreasing sequence $\left(b_{v}\right)_{1}^{\infty}$ such that $\sum_{v=1}^{\infty} a_{v} \sqrt{ } b_{v}=\infty$ and $\sum_{v=1}^{\infty} \alpha_{v} b_{v}<\infty$ and $b_{v+1} / b_{v} \rightarrow 1$ as $\nu \rightarrow \infty$.

Now we construct a function $F$ of the form (4.7) by choosing $m_{v}=2^{2 q_{v}}, k_{v}=4^{q_{v}} b_{v}$ and $a_{v}=k_{v} \omega\left(m_{v}^{-1}\right)$ (we can always adjust $b_{v}$ such that $k_{v}$ becomes odd). Then

$$
\sum_{v=1}^{\infty} a_{v}=\sum_{\nu=1}^{\infty} 4^{q_{v}} \omega\left(m_{v}^{-1}\right) b_{v}=\sum_{1}^{\infty} \alpha_{v} b_{v}<\infty
$$

Thus $F$ is absolutely continuous.

The Fourier-Stieltjes coefficients of $F$ satisfy

$$
\left|d_{n}\right| \geqq \frac{a_{v}}{2 \sqrt{ } k_{v}}-\sum_{\mu=1}^{v-1} \frac{m_{\mu} a_{\mu}}{n \sqrt{ } k_{\mu}}-\sum_{\mu=v+1}^{\infty} \frac{a_{\mu}}{\sqrt{ } k_{\mu}}
$$

for $m_{v-1} \leqq n \leqq m_{v}$. Condition (7.3) enables us to make the same estimations as in $\S 4$ and it follows that $\sum_{1}^{\infty}\left|d_{n}\right| / n$ diverges as $\sum_{1}^{\infty}\left(a_{v} \log m_{v}\right) / \sqrt{ } k_{v}$, i.e. as

$$
\sum_{1}^{\infty} 4^{q_{v}} \omega\left(m_{v}^{-1}\right) \sqrt{ } b_{v}=\sum_{1}^{\infty} \alpha_{v} \sqrt{ } b_{v}
$$

The modulus of continuity of $F$ satisfies the following inequality in $m_{v-1} \leqq n \leqq m_{v}$, obtained from (3.3):

$$
\omega\left(F, n^{-1}\right) \leqq \sum_{\mu=1}^{v-1} \frac{a_{\mu} \cdot m_{\mu}}{n k_{\mu}}+\sum_{\mu=v}^{\infty} \frac{a_{\mu}}{k_{\mu}}+\sum_{\mu=v+1}^{\infty} \frac{2 a_{\mu}}{n} .
$$

Using the fact from (7.3) that $m_{\mu} a_{\mu} k_{\mu}^{-1}$ is increasing exponentially, and $a_{\mu} k_{\mu}^{-1}$ is decreasing exponentially we get

$$
\omega\left(F, n^{-1}\right) \leqq C\left(\frac{a_{v}}{k_{v}}+\frac{m_{v-1} a_{v-1}}{n k_{v-1}}\right)=C\left(\omega\left(m_{v}^{-1}\right)+\frac{m_{v-1}}{n} \omega\left(m_{v-1}^{-1}\right)\right) .
$$


Let $p$ be the natural number, such that $2^{p}<n \leqq 2^{p+1}$. Then we have, since $2^{p} \omega\left(2^{-p}\right)$ is nondecreasing,

$$
\left(m_{v-1} / n\right) \omega\left(m_{v-1}^{-1}\right) \leqq 2^{p+1} \omega\left(2^{-p-1}\right) / n \leqq 2 \omega\left(2^{-p-1}\right) \leqq 2 \omega\left(n^{-1}\right) .
$$

This inequality and the fact that $\omega\left(m_{v}^{-1}\right) \leqq \omega\left(n^{-1}\right)$ and (7.4) yields $\omega\left(F, n^{-1}\right)$ $\leqq C_{1} \omega\left(n^{-1}\right)$. The function $f$, constructed as (4.12) is now a solution to our problem.

COROLlaRY 7.3. If $\varepsilon>0$ and $\omega(h)$ is a nondecreasing function such that $2^{p} \omega\left(2^{-p}\right)$ is nondecreasing and

$$
\sum_{n=1}^{\infty}\left(\omega\left(n^{-1}\right)\right)^{1 / 2} / n(\log \log n)^{(1+\varepsilon) / 2}=\infty
$$

then there exists an absolutely continuous periodic function $f \notin A$ such that $\omega(f, h)$ $\leqq \omega(h)$.

Proof. Cauchy's inequality implies that $\sum_{1}^{\infty}\left(\omega\left(n^{-1}\right) \log n\right) / n=\infty$ and the existence of $f$ follows from Theorem 7.2. Since we may assume that $\omega\left(n^{-1}\right)$ $\leqq(\log n)^{-2}$, we can weaken the corollary by replacing (7.5) with

$$
\sum_{n=1}^{\infty}\left(\omega\left(n^{-1}\right)\right)^{1 / 2} / n \log \left(\omega\left(n^{-1}\right)\right)^{(1+\varepsilon) / 2}=\infty .
$$

This constitutes an improvement of Salem's theorem.

Whether an absolutely continuous function $f$ belongs to $A$ or not obviously depends on its modulus of continuity, $\omega\left(f, n^{-1}\right)$. However, roughly speaking it also depends on how many times $\left|f\left(x+n^{-1}\right)-f(x)\right|$ is of the same order as $\omega\left(f, n^{-1}\right)$. One way of measuring this is to study $\omega_{2}\left(f, n^{-1}\right)$, which leads to Theorem III. Another way is to study the total variation of $f$. We choose the latter and prove below a theorem that is stronger than Theorem I for absolutely continuous functions $f$.

THEOREM 7.4. Let $f$ be an absolutely continuous periodic function and $V_{n}=V_{n}(f)$ $=\int_{E_{n}}\left|f^{\prime}(x)\right| d x$, where $E_{n}=\left\{x ; 0 \leqq x \leqq 1\right.$ and $\left.\left|f^{\prime}(x)\right|>n\right\}$. If $\sum_{1}^{\infty} \sqrt{ }\left(V_{n} \omega\left(f, n^{-1}\right)\right) / n$ $<\infty$ then $f \in A$. Conversely, if $g(n)$ is a nonincreasing function such that $\sqrt{ } n g(n)$ is nondecreasing and $\sum_{1}^{\infty} g(n) / n=\infty$, there exists an absolutely continuous periodic function $f \notin A$ such that $\sqrt{ }\left(V_{n}(f) \omega\left(f, n^{-1}\right)\right) \leqq g(n)$.

Proof. As in $\S 6$ we write

$$
f=g_{N}+h_{N} \quad \text { where } h_{N}=\int_{E_{N} \cap[0, x]} f^{\prime}(t) d t .
$$

We normalize $f$ to have the total variation 1 on $[0,1]$ and obtain from (6.2) and (6.3)

$$
\omega_{2}\left(f, n^{-1}\right) \leqq \sqrt{ } N / n+\sqrt{ }\left(V_{N} \omega\left(h_{N}, n^{-1}\right)\right) / \sqrt{ } n .
$$

Choose $N=n / \log ^{4} n$. Then

$$
\left|\omega\left(h_{N}, n^{-1}\right)-\omega\left(f, n^{-1}\right)\right| \leqq \omega\left(g_{N}, n^{-1}\right) \leqq(\log n)^{-4} .
$$


We see that

$$
\left|\sqrt{ }\left(V_{N} \omega\left(h_{N}, n^{-1}\right)\right)-\sqrt{ }\left(V_{N} \omega\left(f, n^{-1}\right)\right)\right| \leqq(\log n)^{-2} .
$$

Combining this inequality with (7.6) and Theorem III we deduce that $f \in A$ if

$$
\sum_{n=1}^{\infty} \sqrt{ }\left(V_{N} \omega\left(f, n^{-1}\right)\right) / n<\infty, \quad N=n / \log ^{4} n
$$

We want to prove that $V_{N}$ in this formula can be replaced by $V_{n}$. First we show that condition (7.7) implies

$$
\sum_{1}^{\infty} \frac{\sqrt{ }\left(\omega\left(f, n^{-1}\right)\right)}{n \log n}<\infty .
$$

This follows from (7.7) if we observe that $\omega\left(f, n^{-1}\right) \leqq V_{N}+(\log n)^{-4}$. Then

$$
\sum_{n=1}^{\infty} \frac{\sqrt{ }\left(\omega\left(f, n^{-1}\right)\right)}{n \log n} \leqq \sum \frac{\sqrt{ }\left(V_{N} \omega\left(f, n^{-1}\right)\right)}{n}+\sum \frac{\sqrt{ }\left(V_{N}+(\log n)^{-4}\right)}{n \log n}
$$

where the first summation is carried out for those $n$ satisfying $V_{N}>(\log n)^{-2}$. Now (7.8) follows from (7.7). (7.7) is equivalent to

$$
\sum_{n=1}^{\infty} \sqrt{ }\left(V_{2^{p} / p^{4}} \omega\left(f, 2^{-p}\right)\right)<\infty \text { or } \int_{1}^{\infty} p\left(x-4^{2} \log x\right) q(x) d x<\infty
$$

where $\sqrt{ }\left(V_{2^{x}}\right)=p(x)$ and $\sqrt{ }\left(\omega\left(f, 2^{-x}\right)\right)=q(x)$. The convergence of the integral is equivalent to

$$
\int_{1}^{\infty} p(x) q\left(x+4{ }^{2} \log x\right) d x<\infty
$$

But

$$
\begin{aligned}
0 & \leqq\left|\int_{1}^{N} p(x) q(x) d x-\int_{1}^{N} p(x) q\left(x+4{ }^{2} \log x\right) d x\right| \\
& \leqq \int_{1}^{N} q(x) d x-\int_{1}^{N+4 \log N} q(x) d x+10 \int_{1}^{N+5 \log N} \frac{q(x)}{x} d x \\
& \leqq 10 \int_{1}^{\infty} \frac{q(t)}{t} d t .
\end{aligned}
$$

The integral to the right, however, converges as $\sum_{1}^{\infty} \sqrt{ }\left(\omega\left(2^{-n}\right)\right) / n$ or $\sum_{n=1}^{\infty} \sqrt{ }\left(\omega\left(n^{-1}\right)\right) /(n \log n)$. By (7.9) and (7.8) it follows that $\int_{1}^{\infty} p(x) q(x) d x<\infty$, which is equivalent to $\sum_{1}^{\infty} \sqrt{ }\left(V_{n} \omega\left(f, n^{-1}\right)\right) / n<\infty$. This proves the first part of the theorem.

To prove the second part, we make the construction (4.7) of a function $F$. The assumption of the theorem gives $\sum_{1}^{\infty} 5^{q} g\left(2^{5 q}\right)=\infty$. By Lemma 3.3 we can choose a sequence $q_{v}$ such that the sequence $g\left(2^{5^{q_{v}}}\right)$ decreases exponentially with a quotient between $6^{q_{v}-q_{v+1}}$ and $4^{q_{v}-q_{v+1}}$ and such that $\sum_{1}^{\infty} 5^{q_{v}} g\left(2^{5 q_{v}}\right)=\infty$. 
We choose $a_{v}=3^{-v}, m_{v}=2^{5 a_{v}}$ and $k_{v}=\left(3^{v} g\left(m_{v}\right)\right)^{-2}$. As before, we estimate the Fourier-Stieltjes coefficients of $F, d_{n}$, and get

$$
\sum_{n=1}^{\infty} \frac{\left|d_{n}\right|}{n}>C \sum_{v=1}^{\infty} \frac{a_{v} \log m_{v}}{\sqrt{ } k_{v}}=\sum_{v=1}^{\infty} 5^{a_{v}} g\left(2^{5 a_{v}}\right)=\infty .
$$

For $m_{v-1} \leqq n \leqq m_{v}$ we have $V_{n}(F)<\sum_{\mu=v}^{\infty} a_{\mu} \leqq C_{1} a_{v}$ and

$$
\omega\left(F, n^{-1}\right) \leqq \sum_{\mu=1}^{v=1} \frac{m_{\mu} a_{\mu}}{n k_{\mu}}+\sum_{\mu=v}^{\infty} \frac{a_{\mu}}{k_{\mu}}+\sum_{\mu=v+1}^{\infty} \frac{a_{\mu}}{n} .
$$

These three series are dominated by their first, last and first term, respectively, and we obtain

$$
V_{n}(F) \cdot \omega\left(F, n^{-1}\right) \leqq C_{2}\left(a_{v}^{2} / k_{v}+m_{v-1} a_{v-1}^{2} / n k_{v-1}\right)
$$

Thus

$$
\sqrt{ }\left(V_{n}(F) \omega\left(F, n^{-1}\right)\right) \leqq \sqrt{ } C_{2}\left(g\left(m_{v}\right)+\sqrt{ }\left(m_{v-1} / n\right) g\left(m_{v-1}\right)\right) .
$$

The assumption that $\sqrt{ } n g(n)$ is nondecreasing gives

$$
\sqrt{ }\left(V_{n} \omega\left(F, n^{-1}\right)\right) \leqq C_{3} g(n) .
$$

It is obvious that the function $f$ constructed as in (4.12) also has the property (7.10) and does not belong to $A$. This completes the proof of Theorem 7.4.

8. Conditions on $\omega_{p}, p>1$. In this section we shall give two theorems, with conditions on $\omega_{p}, p>1$. For $p=2$ they coincide with Theorem III.

THEOREM 8.1. ( $p \geqq 2)$. Let $f$ be a periodic function, absolutely continuous and satisfying

$$
\sum_{n=1}^{\infty}\left(\omega_{p}\left(f, n^{-1}\right)\right)^{p / 2(p-1)} V_{n}^{(p-2) / 2(p-1)} n^{-(2 p-3) / 2(p-1)}<\infty .
$$

Then $f \in A$. Conversely, if $g$ is a nondecreasing function, satisfying

(a) $\left(2^{q} g\left(2^{q}\right)\right)_{1}^{\infty}$ is a nondecreasing sequence,

(b) $\left(2^{p}\left(g\left(2^{p}\right)\right)^{2(p-1)}\right)_{1}^{\infty}$ is a nonincreasing sequence,

(c) $\sum_{1}^{\infty} g(n) \cdot n^{-(2 p-3) / 2(p-1)}<\infty$,

then there exists an absolutely continuous periodic function $f \notin A$ such that

$$
\omega_{p}\left(f, n^{-1}\right)^{p / 2(p-1)}\left(V_{n}(f)\right)^{(p-2) / 2(p-1)} \leqq g(n) .
$$

Proof. For the first part of the theorem we apply Hölder's inequality to the integral

$$
\int_{0}^{1}|f(x+t)-f(x)|^{2} d x=\int_{0}^{1}|f(x+t)-f(x)|^{p /(p-1)}|f(x+t)-f(x)|^{(p-2) /(p-1)} d x .
$$

Then we obtain

$$
\omega_{2}^{2}\left(f, n^{-1}\right) \leqq\left(\omega_{p}\left(f, n^{-1}\right)\right)^{p /(p-1)} \omega_{1}\left(f, n^{-1}\right)^{(p-2) /(p-1)} .
$$


Using the fact (2.5) that $\omega_{1}\left(f, n^{-1}\right) \leqq V_{f} / n$, we find

$$
\omega_{2}\left(f, n^{-1}\right) / \sqrt{ } n \leqq \omega_{p}\left(f, n^{-1}\right)^{p / 2(p-1)} V_{f}^{(p-2) / 2(p-1)} n^{-(2 p-3) / 2(p-1)} .
$$

The same method as in Theorem 7.4 now makes it possible to replace $V_{f}$ by $V_{n}(f)$. The theorem now follows from Theorem III.

The second part is proved by constructing a function $F$ as in the second part of Theorem 7.4. We put $m_{v}=2^{5 q_{v}}, a_{v}=3^{-v}$ and $a_{v} / \sqrt{ } k_{v}=m_{v}^{1 / 2(p-1)}\left(g\left(m_{v}\right)\right)^{p / 2(p-1)}$ and the estimations (3.5) will give us the desired result.

REMARK I. We observe that the estimation (8.1) has the consequence that

$$
\sum_{n=1}^{\infty}\left(\omega\left(f, n^{-1}\right)\right)^{p / 2(p-1)} n^{-(2 p-3) / 2(p-1)}<\infty
$$

implies that $f \in A$ if $f$ is continuous, periodic and of bounded variation.

REMARK II. Theorem 8.1 is true even for $p=\infty$. It is then Theorem 7.4.

For $1 \leqq p \leqq 2$ we have the following theorem.

THEOREM 8.2. Let $f$ be a periodic function, absolutely continuous and such that

$$
\sum_{n=1}^{\infty}\left(\omega_{p}\left(f, n^{-1}\right)\right)^{p / 2} \omega\left(f, n^{-1}\right)^{1-p / 2} / \sqrt{ } n<\infty .
$$

Then $f \in A$. Conversely, if $g$ is a nonincreasing function, satisfying

(a) $\left(2^{p} g\left(2^{p}\right)\right)_{1}^{\infty}$ is a nondecreasing sequence,

(b) $\left(2^{p / 2} g\left(2^{p}\right)\right)_{1}^{\infty}$ is a nonincreasing sequence,

(c) $\sum g(n) / \sqrt{ } n=\infty$,

then there exists an absolutely continuous, periodic function $f \notin A$, such that

$$
\omega_{p}\left(f, n^{-1}\right)^{p / 2} \omega\left(f, n^{-1}\right)^{1-p / 2} \leqq g(n) .
$$

Proof. The first part follows from Theorem III and the second part is proved similarly to Theorem 4.1.

REMARK. The positive part of Theorem 8.2 is a special case of a theorem of $\mathbf{M}$. and S. Izumi [2].

\section{Necessary and sufficient conditions.}

THEOREM 9.1. Let $f$ be a realvalued absolutely continuous function, with period 1 and

$$
E_{n}=\left\{x ; 0 \leqq x \leqq 1 \text { and }\left|f^{\prime}(x)\right|>n\right\}
$$

\section{Suppose that}

(a) $f^{\prime}(x)$ is uniformly bounded below (above),

(b) the diameter of $E_{n}, d\left(E_{n}\right)$, is $O(1 / n)$.

Then a necessary and sufficient condition that $f \in A$ is

$$
\int_{0}^{1}\left|f^{\prime}\right| \log ^{+}\left|f^{\prime}\right| d x<\infty
$$


Proof. Without loss of generality, we may assume that the total variation of $f$, $V_{f}$, is 1 and $f(0)=0$. We also assume that $d\left(E_{n}\right) \leqq 1 / 4 n$. Put $f(x)=g_{N}(x)+h_{N}(x)$, where

$$
h_{N}(x)=\int_{E_{N} \cap[0, x]} f^{\prime}(t) d t+A_{N} x=h_{N}^{*}(x)+A_{N} x,
$$

and $A_{N}$ is a constant chosen such that $h_{N}$ is periodic.

Obviously

$$
A_{N} \leqq \int_{E_{N}}\left|f^{\prime}(x)\right| d x=V_{N} \rightarrow 0 \text { as } N \rightarrow \infty
$$

Then

$$
g_{N}(x)=\int_{C\left(E_{N}\right) \cap[0, x]} f^{\prime}(t) d t-A_{N} x=g_{N}^{*}-A_{N} x .
$$

The Fourier coefficients of $f, g_{N}$ and $h_{N}$ are denoted by $a_{n}, a_{n}\left(g_{N}\right)$ and $a_{n}\left(h_{N}\right)$ respectively. Then by (1.4)

But

$$
\sum_{n=2^{p-1}}^{2^{p}}\left|a_{n}\left(g_{N}\right)\right| \leqq 2^{p / 2} \omega_{2}\left(g_{N}, 2^{-p-1}\right) .
$$

$$
\omega_{2}\left(g_{N}, 2^{-p-1}\right) \leqq \omega_{2}\left(g_{N}^{*}, 2^{-p-1}\right)+\omega_{2}\left(A_{N} x, 2^{-p-1}\right) \leqq \sqrt{ } N / 2^{p+1}+A_{N} / 2^{p+1}
$$

for any $N>0$. We choose $N=2^{p} / p^{4}$ and obtain

$$
\sum_{n=2^{p-1}}^{2^{p}}\left|a_{n}\left(g_{N}\right)\right| \leqq \frac{1}{2 p^{2}}+\frac{A_{N}}{2^{p / 2}}
$$

Hence,

$$
\sum_{n=2^{p-1}}^{2^{p}}\left|a_{n}\left(g_{N}\right)\right| \leqq \frac{1}{p^{2}} \text { for } p \geqq p_{0}
$$

The Fourier coefficients of $h_{N}$ satisfy

$$
\begin{aligned}
a_{n}\left(h_{N}\right) & =-\frac{1}{2 \pi i n} \int_{0}^{1} h_{N}^{\prime}(x) e^{2 \pi i n x} d x=-\frac{1}{2 \pi i n} \int_{0}^{1} h_{N}^{*^{\prime}}(x) e^{2 \pi i n x} d x \\
& =-\frac{1}{2 \pi i n} \int_{E_{N}} f^{\prime}(x) e^{2 \pi i n x} d x .
\end{aligned}
$$

Since $E_{n} \supset E_{n+1}, n=1,2, \ldots$, we have

$$
\int_{E_{2^{p} / p^{4}}} f^{\prime}(x) e^{2 \pi i n x} d x=\int_{E_{2^{p}}} f^{\prime}(x) e^{2 \pi i n x} d x+\int_{E_{2^{p} / p^{4} \backslash E_{2}^{p}}} f^{\prime}(x) e^{2 \pi i n x} d x .
$$

From assumption (a) it follows, for sufficiently large values of $p$, that the inequality $f^{\prime}(x)>2^{p}>0$ holds on $E_{2^{p}}$. Since $d\left(E_{2^{p}}\right) \leqq 2^{-(p+2)}$, we find, for $n \leqq 2^{p}$,

$$
\left|\int_{E_{2^{p}}} f^{\prime}(x) e^{2 \pi i n x} d x\right| \geqq \frac{1}{\sqrt{ } 2} \int_{E_{2^{p}}} f^{\prime}(x) d x=V_{2^{p}} / \sqrt{ } 2 .
$$


As before, we use the notation

$$
V_{n}=\int_{E_{n}}\left|f^{\prime}(x)\right| d x
$$

We note that

$$
\left|\int_{E_{2^{p / p^{4}} \mid E_{2}}} f^{\prime}(x) e^{2 \pi i n x} d x\right| \leqq V_{2^{p} / p^{4}}-V_{2^{p}} .
$$

Combining (9.1)-(9.5) we find

$$
\begin{aligned}
\sum_{2^{p-1}}^{2^{p}}\left|a_{n}\right| & \geqq \sum_{2^{p-1}}^{2^{p}}\left|a_{n}\left(h_{N}\right)\right|-\sum_{2^{p-1}}^{2^{p}}\left|a_{n}\left(g_{N}\right)\right| \\
& \geqq\left(V_{2^{p}}(1+1 / \sqrt{ } 2)-V_{2^{p} / p^{4}}\right)\left(\sum_{2^{p-1}}^{2^{p}} \frac{1}{2 \pi n}\right)-\frac{1}{p^{2}},
\end{aligned}
$$

for sufficiently large values of $p$. It follows that

$$
\sum_{n=1}^{\infty}\left|a_{n}\right|=\infty \quad \text { if } \sum_{p=1}^{\infty}\left((1+1 / \sqrt{ } 2) V_{2^{p}}-V_{2^{p} / p^{4}}\right)=\infty
$$

The function $v(x)=V_{2^{x}}$ is nonincreasing and positive. Thus

$$
\begin{aligned}
\sum_{p_{1}}^{P} V_{2^{p} / p^{4}} & \leqq \int_{p_{1}}^{P} v\left(x-4{ }^{2} \log x\right) d x+v\left(p_{1}\right) \\
& \leqq v\left(p_{1}\right)+\frac{3}{2} \int_{P_{1}-42 \log p_{1}}^{p_{1}} v(t) d t+\frac{3}{2} \int_{p_{1}}^{P} v(t) d t,
\end{aligned}
$$

if $p_{1}>20$. Also

$$
\int_{p_{1}}^{P} v(t) d t \leqq \sum_{p_{1}}^{P} v(p)=\sum_{p_{1}}^{P} V_{2^{p}}
$$

(9.7) and (9.8) yield

$$
\sum_{p_{1}}^{P}\left(\left(1+\frac{1}{\sqrt{ } 2}\right) V_{2^{p}}-V_{2^{p} / p^{4}}\right) \geqq\left(1+\frac{1}{\sqrt{ } 2}-\frac{3}{2}\right) \sum_{p_{1}}^{P} V_{2^{p}}-C\left(P_{1}\right) .
$$

From (9.6) we now deduce that $\sum_{1}^{\infty}\left|a_{n}\right|$ diverges if $\sum_{1}^{\infty} V_{2^{p}}$ diverges. By Lemma 6.1 this happens if and only if

$$
\int_{0}^{1}\left|f^{\prime}\right| \log ^{+}\left|f^{\prime}\right| d x=\infty
$$

This proves the necessity part of the theorem.

The sufficiency follows from Theorem II but can also easily be deduced from the estimation $\sum_{2^{p-1}}^{2^{P}}\left|a_{n}\left(h_{N}\right)\right| \leqq V_{2^{p} / p^{4}}$ combined with (9.7) and (9.8).

In the proof of the theorem, we have for convenience used the assumption that the diameter $d\left(E_{n}\right) \leqq 1 / 4 n$. To prove the theorem with $1 / 4 n$ replaced by $O(1 / n)$ we replace the summations $\sum_{2^{p-1}}^{2^{p}}\left|a_{n}\right|$ by $\sum_{2^{p-k-1}}^{2^{p-k}}\left|a_{n}\right|$ for a suitable fixed number $k$. 
REMARK 1. The condition that $f$ is realvalued can be removed if we change condition (a) to

$\left(\mathrm{a}^{\prime}\right)(\operatorname{Re}(f))^{\prime}$ and $(\operatorname{Im}(f))^{\prime}$ are bounded from below or above.

REMARK 2. If $f$ has the properties (a) and (b) of Theorem 9.1 in a neighborhood of a finite number of points in (0.2) and belongs locally to $A$ elsewhere, the condition

$$
\int_{0}^{1}\left|f^{\prime}\right| \log { }^{+}\left|f^{\prime}\right| d x
$$

is still necessary and sufficient. This is seen if we isolate the critical points by multiplying $f$ with a suitable function in $A$, which is $\equiv 1$ in a neighborhood of a critical point and 0 outside another neighborhood and has bounded derivatives.

REMARK 3. The condition that $f^{\prime}$ is bounded below (above) can also be weakened. The property we use is

$$
\left|\int_{E_{n}} f^{\prime} e^{2 \pi i n x} d x\right| / \int_{E_{n}}\left|f^{\prime}(x)\right| d x \geqq C>0 \text { for } n>n_{0} .
$$

See also Theorem 9.2 below.

REMARK 4. To see that Theorem IV of Zygmund is a special case of Theorem 9.1 we simply observe that for any function $f$, such that $f^{\prime}$ is nonincreasing in $0<x<\delta$ and $f(0)=0$, we have $f(x)=f(x)-f(0) \geqq x f^{\prime}(x)$. Thus $f^{\prime}(1 / n) \leqq n$, for $n>n_{0}$. It follows that the conditions of Theorem 9.1 are satisfied in a neighborhood of 0 if $f$ is odd, $f^{\prime}$ is nonincreasing in $(0, \delta)$ and bounded in $[\delta, 1-\delta]$.

COROLlaRY. Under the assumptions of Theorem 9.1, a necessary and sufficient condition that $f \in A$ is

$$
\sum_{n=1}^{\infty} \omega_{2}\left(f, n^{-1}\right) / \sqrt{ } n<\infty
$$

Proof. The sufficiency follows from Theorem III and the necessity from the fact, proved in $\S 6$, that $(9.9)$ is implied by the condition

$$
\int_{0}^{1}\left|f^{\prime}\right| \log { }^{+}\left|f^{\prime}\right| d x<\infty
$$

In fact we may, if we wish, change $\omega_{2}$ to $\omega_{2}^{*}(\S 5)$ in (9.9).

REMARK. It is possible to use a simple construction, based on Theorem 9.1 , to prove Theorem 4.1 with somewhat stronger assumptions. However, we need the function constructed in $\S 4$ on several other occasions, particularly in $\S 7$. We have therefore preferred to use mainly the same type of counterexamples throughout the paper.

In the case where $f^{\prime}$ is not bounded below (above), for example if $f$ is even, we use a similar method to prove the following theorem. 
THEOREM 9.2. If $f$ has period $1, f^{\prime}$ is bounded in $(\delta, 1-\delta)$ for every $\delta>0, d\left(E_{n}\right)$ $=O(1 / n)$ and

$$
\int_{0}^{1}\left|f^{\prime}\right| \log { }^{+} \log ^{+}\left|f^{\prime}\right| d x<\infty
$$

then $f \in A$ if and only if

$$
\sum_{p=1}^{\infty}\left|\int_{E_{2^{p}}} f^{\prime}(x) d x\right|<\infty
$$

Remark. For even functions $f$ the terms of the series (9.11) are all zero, whence (9.10) in this case is a sufficient condition that $f \in A$.

Proof of Theorem 9.2. We consider only positive values of $n$; the negative integers are treated similarly. For the sake of convenience we may as before suppose that $d\left(E_{n}\right) \leqq 1 / 4 n$. Using (9.2) and the fact that $\left|e^{i \delta}-1\right|<2 \delta$, if $\delta<2$, we find, for $N=2^{p} / p^{4}$ and $2^{p-1} \leqq n \leqq 2^{p}$,

$$
\begin{gathered}
\left|a_{n}\left(h_{N}\right)+\frac{1}{2 \pi i n} \int_{E_{2^{p}}} f^{\prime}(x) e^{2 \pi i n x} d x\right| \leqq \frac{1}{2 \pi n}\left(V_{2^{p} / p^{4}}-V_{2^{p}}\right), \\
\left|\frac{1}{2 \pi i n} \int_{E_{2^{p}}} f^{\prime}(x) e^{2 \pi i n x} d x-\frac{1}{2 \pi i n} \int_{E_{2^{p}}} f^{\prime}(x) d x\right| \leqq 2 \int_{E_{2^{p}}}|x|\left|f^{\prime}(x)\right| d x .
\end{gathered}
$$

The theorem thus is proved if we can show that

$$
\sum_{p=1}^{\infty}\left(V_{2^{p} / p^{4}}-V_{2^{p}}\right)<\infty
$$

and

$$
\sum_{p=1}^{\infty} 2^{p} \int_{E_{2^{p}}}|x|\left|f^{\prime}(x)\right| d x<\infty
$$

We put $V_{2^{x}}=v(x)$ and find

Thus

$$
\sum_{10}^{P} V_{2^{p} / p^{4}} \leqq C_{1}+\int_{10}^{P} v\left(x-4^{2} \log x\right) d x \leqq C_{2}+\int_{10}^{P} \frac{v(t) d t}{1-8 / t}
$$

$$
\int_{10}^{P}\left(v\left(x-4{ }^{2} \log x\right)-v(x)\right) d x \leqq C_{3}+40 \int_{10}^{\infty}(v(t) / t) d t<\infty .
$$

The last inequality follows from the fact that

$$
\int_{1}^{\infty}\left|f^{\prime}\right| \log ^{+} \log ^{+}\left|f^{\prime}\right| d x<\infty \Leftrightarrow \sum \frac{V_{2^{p}}}{p}<\infty .
$$

(Compare Lemma 6.1.) This proves (9.12).

To prove (9.13) we observe that

$$
2^{P} \int_{E_{2} P}|x|\left|f^{\prime}(x)\right| d x \leqq 2^{P} \int_{0}^{2^{-P}}|x|\left|f^{\prime}(x)\right| d x+2^{P} \int_{-2^{-p}}^{0}|x|\left|f^{\prime}(x)\right| d x .
$$

These two integrals are treated similarly; we pick the first.

$$
2^{P} \int_{0}^{2^{-P}}|x|\left|f^{\prime}(x)\right| d x \leqq 2^{P} \sum_{q=p}^{\infty} 2^{-q} \int_{2^{-q-1}}^{2^{-q}}\left|f^{\prime}(x)\right| d x .
$$


We reverse the order of summation and find

$$
\begin{aligned}
\sum_{1}^{\infty} 2^{P} \int_{0}^{2^{-P}}|x|\left|f^{\prime}(x)\right| d x & \leqq \sum_{q=1}^{\infty} 2^{-q} \int_{2^{-q-1}}^{2^{-q}}\left|f^{\prime}(x)\right| d x \sum_{p=1}^{q} 2^{P} \\
& \leqq 2 \int_{0}^{1}\left|f^{\prime}(x)\right| d x<\infty .
\end{aligned}
$$

This concludes the proof of the theorem.

If $f$ is subject to more restrictive conditions than in Theorem 9.2, a stronger, sufficient condition can be obtained. It has been pointed out by Zygmund in [8] and generalized by Sz.-Nagy in [4].

THEOREM 9.3 (ZyGMUND). Let $f$ have period 1 and be even. If, for some $\delta>0$, $f$ is convex in $(0, \delta)$ and $f^{\prime}$ is bounded in $(\delta, 1-\delta)$, then $f \in A$.

Corollary. Let $\omega(h), h>0$, be any positive function tending to zero with $h$. Then there exists an absolutely continuous function $f \in A$ such that $\omega(f, h) \geqq \omega(h), h \leqq \delta$.

Proof. Choose $f$ as a convex majorant of $\omega(h)$ in $(0, \delta), f(0)=0$, make it even in $[-\delta, \delta]$ and define it linearly in $[\delta, 1-\delta]$. Then we have a function satisfying the conditions of Theorem 9.3 and obviously $\omega(f, h)=f(h) \geqq \omega(h)$.

Compare [1, p. 178].

REMARK. Using Theorems 9.1 and 9.2 or 9.3 it is easy to find examples of even functions belonging to $A$ such that the corresponding odd function does not belong to $A$. Kahane has in [3] proved that there exists odd functions (not of bounded variation) belonging to $A$ such that the corresponding even function does not belong to $A$.

\section{REFERENCES}

1. N. K. Bari, Trigonometric series, Fizmatgiz, Moscow, 1961; English transl., Macmillan, New York; Pergamon Press, Oxford, 1964. MR 23 \#A3411; MR 30 \#1347.

2. M. Izumi and S. Izumi, On absolute convergence of Fourier series, Ark. Mat. 7 (1967), 177184. MR 36 \#4247.

3. J.-P. Kahane, Sur certaines classes de séries de Fourier absolument convergentes, J. Math. Pures Appl. (9) 35 (1956), 249-259. MR 20 \#4149.

4. B. Sz.-Nagy, Séries et intégrales de Fourier des fonctions monotones non bornées, Acta Univ. Szeged. Sect. Sci. Math. 13 (1949), 118-135. MR 11, 656.

5. R. Salem, On a theorem of Zygmund, Duke Math. J. 10 (1943), 23-31. MR 4, 156.

6. O. Szasz, Fourier series and mean moduli of continuity, Trans. Amer. Math. Soc. 42 (1937), 366-395.

7. I. Wik, Extrapolation of absolutely convergent Fourier series by identically zero, Ark. Mat. 6 (1965), 65-76. MR 33 \#4585.

8. A. Zygmund, Sur les fonctions conjuguées, Fund. Math. 13 (1929), 284-303.

9. —-, Trigonometrical series, 2nd rev. ed., Cambridge Univ. Press, New York, 1959. MR 21 \#6498.

University of Washington, Seattle, Washington 98105

UNIVERSITY OF UMEÅ, UMEÅ, SWEDEN 